\title{
A Method to Generate Disaster-Damage Map by Using 3D photometry and Crowd Sourcing
}

\author{
Koyo Kobayashi \\ University of Tsukuba \\ College of Engineering Systems \\ Tsukuba, Japan \\ e-mail: s1311111@u.tsukuba.ac.jp
}

\author{
Yoshinari Kameda \\ University of Tsukuba \\ Center for Computational Sciences \\ Tsukuba, Japan \\ e-mail:kameda@ccs.tsukuba.ac.jp
}

\author{
Hidehiko Shishido \\ University of Tsukuba \\ Center for Computational Sciences \\ Tsukuba, Japan \\ e-mail: shishido@ccs.tsukuba.ac.jp \\ Itaru Kitahara \\ University of Tsukuba \\ Center for Computational Sciences \\ Tsukuba, Japan \\ e-mail: kitahara@ccs.tsukuba.ac.jp
}

\begin{abstract}
With to the spread of the Internet and mobile devices, information related to disaster areas is being collected through the Internet. To grasp the degree of damage in a disaster situation, the use of crowdsourcing for collecting the trivial efforts (micro tasks) of an enormous number of users (workers) on the Internet has been drawing attention as a mean of quickly solving problems. However, the information gathered from the Internet is huge and diverse, so it is difficult to formulate it as a crowdsourcing task. In this paper, we propose a conversion platform for the images of the site photographed by various users as information of the disaster site, integrating them into a single map using 3D image processing, and providing it to crowdsourcing as a micro task.
\end{abstract}

Keywords-component; crowdsourcing; $3 D$ reconstruction; multi-view image; damage state grasp

\section{INTRODUCTION}

With the development of Internet tools represented by SNSs, it is becoming possible to directly collect the information regarding the situation of the disaster is occurring [1]. However, since data received from an unspecified majority is massive and fragmentary, it is still not realistic to check all the data or to grasp the situation of the site automatically [2]. In crowdsourcing, many users connected to the Internet can handle large amounts of data. Therefore, it is a promising means of processing that enables us to extract useful information contained in incomplete data by using human advanced judgment ability. When designing a crowdsourcing implementation, it is essential to organize a large amount of data into fragments that can be handled by the user or to modelize it (making appropriate micro tasks). In this paper, we propose a method of treating multi - view image data collected at the time of a disaster as a large amount of data and generating a micro task which is necessary for efficient crowdsourcing of grasp of the damage situation by $3 \mathrm{D}$ reconstruction using the crowdsourcing platform.

\section{RELATES WORK}

\section{A. Disaster damage assessmen using crowdsourcing}

Inoguchi has used crowdsourcing to assist in damage assessment as one of the projects on this platform [3][4]. In this crowdsourcing micro task, they present the image of the affected area cropped from the aerial photograph to the workers and have them classify it under one of the several predefined classes, to judge the disaster situation. However, since the information to be presented is a two-dimensional image, it is known that there is a limit to how well the situation can be determined by the operator. For example, Fig. 1 shows an example image that was actually used in micro tasks. As is apparent, it is difficult to confirm the state of the sides of the structure in the image photographed from immediately above, and it is difficult to judge whether or not the building has collapsed.

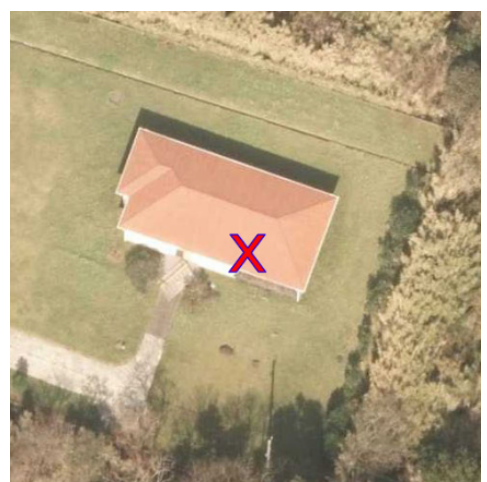

Figure 1. Aerial photograph by Inotuchi's micro task.

\section{B. Structure from Motion}

There have been many studies on SfM (Structure from Motion), in which one estimates the 3D shape data of a photographing space and the position and orientation of a 


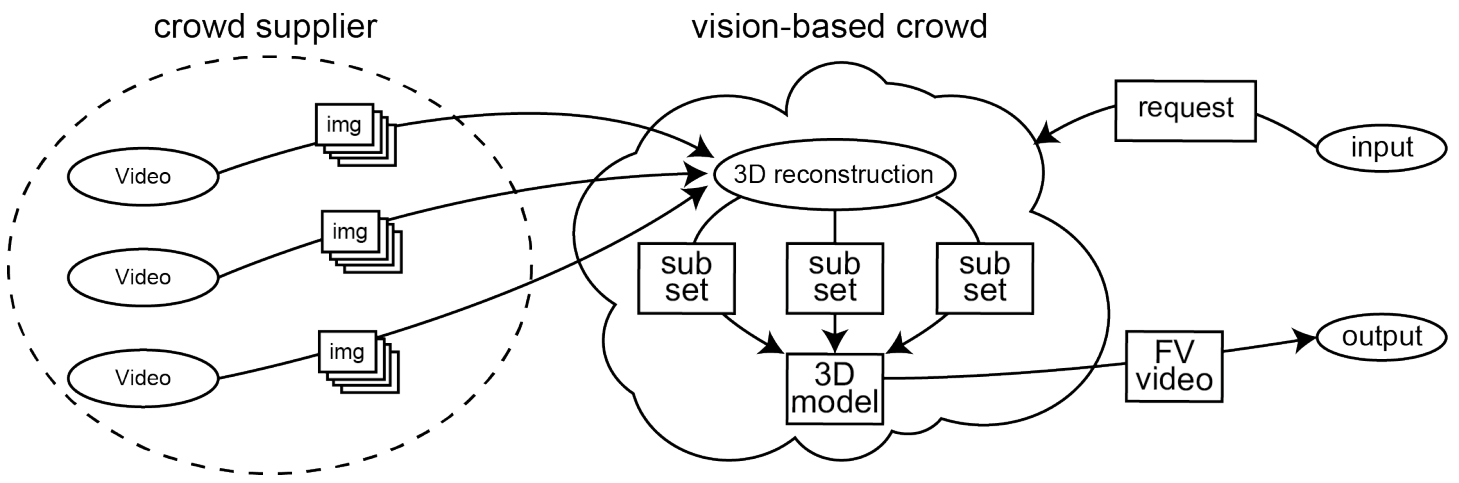

Figure 2. Flow of the proposed method.

photographing camera using corresponding point information detected on a multi-view point image. Agarwal et al. have proposed a method of realizing the $3 \mathrm{D}$ reconstruction of the whole city by using a huge group of images from an Internet photo sharing site [5]. It uses shooting position information recorded in the Exif data area of the JPEG image, by doing so, the image retrieval process is improved.

\section{OVERVIEW of The Proposed Method}

Fig. 2 shows the workflow of the proposed method. We will conduct crowdsourcing to publicly invite collaborators and gather multi-view images of disaster area on the Web. By doing so, we gather information necessary for judging the disaster situation (crowd supplier).

Such crowdsourcing activity is often completed too late, even if it is started at the time of a disaster. To prepare for a sudden disaster, letting the local people constantly operate on a topography grasping task may allow a better preparation and understanding of the terrain. SfM is applied to the collected multi-view image, and a 3D model around the disaster site is generated. From this 3D model, micro tasks for judging the collapse of houses in the disaster site are generated and provided to the crowd worker to judge the disaster situation. As a result, the comprehensive map of the disaster situation which summarizes the result is generated. In this micro task, to realize the judgment of the disaster situation easily and with high accuracy, we present a free viewpoint image which can be observed by the subject in multiple ways.

Crowd supplier is a crowdsourcing based on the is a micro task that involves capturing images from multiple viewpoints using handheld terminals. The crowd worker detects and identifies the collapsed houses and damaged areas in the generated video. It is crowdsourcing based in micro tasks that are aimed at collecting the situation of the damaged area.

The Vison-Based Crowd hands over two crowdsourcing calls for image (video) of the disaster site to the Crowd Supplier, based on the disaster information from the disaster prevention agency. A group of multi-view point images is sent from the Crowd Supplier, then 3D restoration processing is applied to generate a 3D model of a disaster area. A video is produced in which the viewpoint draws a circle around the point where you want to know the damage situation by using free viewpoint video technology, and the damage situation is judged at that point by the crowd worker. If the Crowd Worker encounters an error or inaccuracy in the information related to the location, he will inform the Vision-Based Crowd in order to correct the information of the site of disaster, and this improves the judgment accuracy.

\section{3D RECONSTRUCTION AND ADDITION OF GIS INFORMATION}

We will publicly invite users to provide multi-view images of disaster areas by means of crowdsourcing. The resulting multi-view image is assumed to be an image in which inaccuracy information is included in the Exif. By including the positional information in the image, the amount of processing required for restoration can be reduced. Also, in order to generate free viewpoint images of buildings from multi-view images, the coordinates of those buildings are required. GIS (Geographical Information System) offers various geographical information. In order to improve the address database, GIS information is added to the 3D model from the GPS data included in Exif. Fig. 3 shows an example in which a 3D model is linked with a satellite map made from GIS information, and by doing so it can be confirmed that the coordinates of the $3 \mathrm{D}$ model correspond to those of the map.

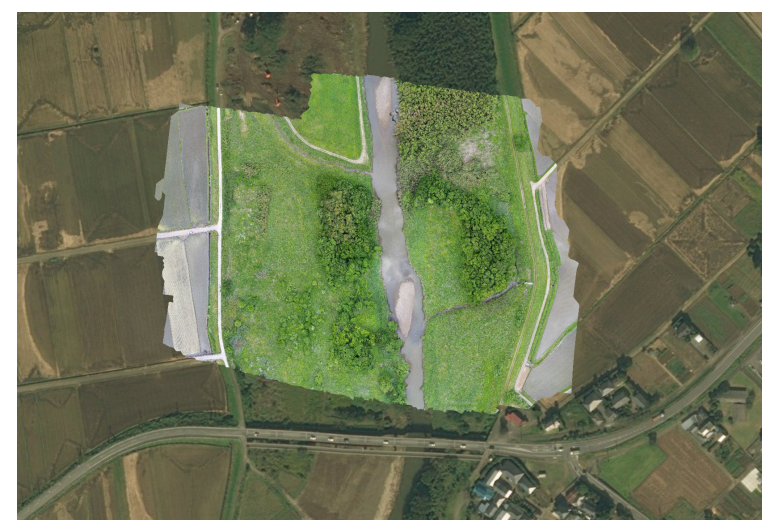

Figure 3. 3D model with GIS information added. 


\section{Generation of FreE Viewpoint Video By 3D MODEL}

We generate a micro task that makes it possible to judge the possible collapse for each building in the disaster area, by means of the resulting 3D model with the corresponding GIS information added from the address database. The micro task should be handled easily and quickly by the worker, but the 3D model of the entire affected site is not suitable for the micro task because it contains a lot of information. Therefore, the worker is presented with a free viewpoint video that focuses on the 3D model of a residence. The free multi-view image is generated by a camera rig that rotates around a point centered on the coordinates of the building, of which address is acquired from the address database. Fig. 4 shows the result of the camerawork of the free viewpoint image centering on the building. Fig. 5 shows an example of a free viewpoint image actually generated from a 3D model. With this camera work, since it is possible to see the roof and all sides, the condition of the structure can be judged with high accuracy, including whether it has collapsed or not.

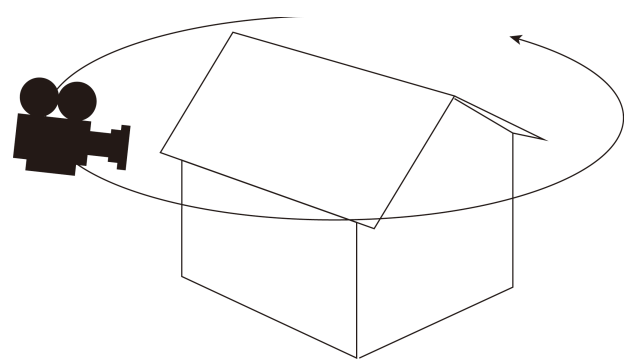

Figure 4. Image of the camera of free viewpoint video.

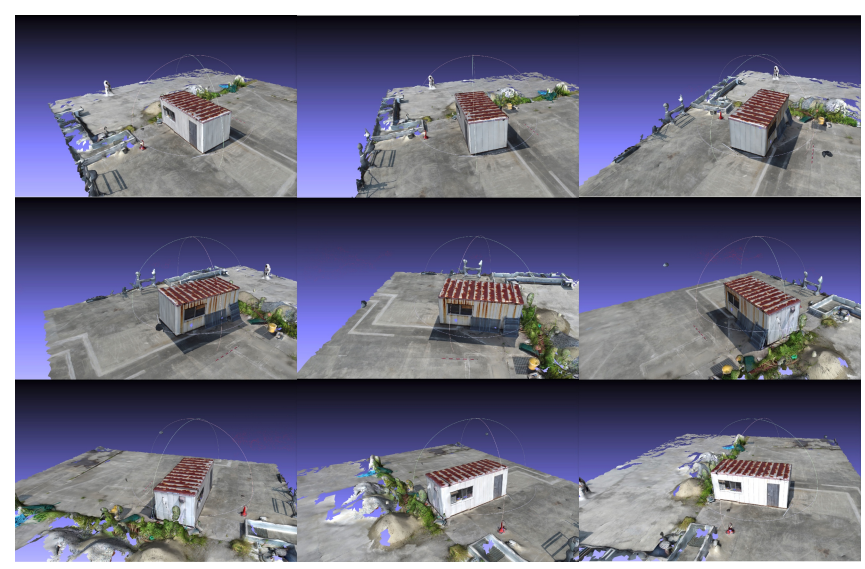

Figure 5. Examples of free viewpoint images.

\section{Micro TASK PROVISION FOR CROWDSOURCING}

A micro task that classifies the state of possible collapse houses are generated from the free viewpoint video generated with the 3D model. The micro task is provided for the crowd sourcing to allow the map generation of the disaster zone.

\section{CONCLUSIONS}

In this research, we propose a generation process of micro task which are necessary to efficiently generate an accurate map of the disaster situation using 3D image processing and crowdsourcing. In the future, we plan to conduct research on camerawork for free viewpoint video, which is the most suitable for judging the collapse of houses.

\section{ACKNOWLEDGMENT}

This work was supported by JST CREST Grant Number JPMJCR16E3, Japan.

\section{REFERENCES}

[1] Liliya I. Besaleva, Alfred C. Weaver, "Applications of Social Networks and Crowdsourcing for Disaster Management Improvement", Computer, pp. 44-57, May 2016.

[2] Jason D. Renwick, Levente J. Klein, and Hendrik F. Hamann, "Dronebased reconstruction for 3D geospatial data processing," 2016 IEEE 3rd World Forum on Internet of Things (WF-IoT), pp. 729-734, December 2016 .

[3] Atsuyuki Morishima, Norihide Shinagawa, Tomomi Mitsuishi, Hideto Aoki and Shun Fukusumi "CyLog/Crowd4U: A Declarative Platform for Complex Data-centric Crowdsourcing", Proceedings of the VLDB Endowment Volume 5 Issue 12, pp. 1918-1921, August 2012

[4] Munenari Inoguchi et al., "MIND: Micro-volunteering for Help in Natural Disasters", "http://crowd4u.org/en/projects"

[5] Sameer Agarwal, Noah Snavely, Ian Simon, Steven M. Seitz and Richard Szeliski, "Reconstructing Rome", IEEE Computer, pp. 40-47, June, 2010 